\title{
Mapping of Olfactory Memory Circuits: Region-Specific c-fos Activation After Odor-Reward Associative Learning or After Its Retrieval
}

\author{
Sophie Tronel and Susan J. Sara ${ }^{1}$ \\ Neurobiologie des Processus Adaptatifs Centre National de la Recherche Scientifique, Unite Mixte de Recherche 7102, Laboratoire de \\ Neuromodulation et Processus Mnésiques, Université P \& M Curie, 75005 Paris, France
}

\begin{abstract}
Although there is growing knowledge about intracellular mechanisms underlying neuronal plasticity and memory consolidation and reconsolidation after retrieval, information concerning the interaction among brain areas during formation and retrieval of memory is relatively sparse and fragmented. Addressing this question requires simultaneous monitoring of activity in multiple brain regions during learning, the post-acquisition consolidation period, and retrieval and subsequent reconsolidation. Immunoreaction to the immediate early gene $c$-fos is a powerful tool to mark neuronal activation of specific populations of neurons. Using this method, we are able to report, for the first time, post-training activation of a network of closely related brain regions, particularly in the frontal cortex and the basolateral amygdala (BLA), that is specific to the learning of an odor-reward association. On the other hand, retrieval of a well-established associative memory trace does not seem to differentially activate the same regions. The amygdala, in particular, is not engaged after retrieval, whereas the lateral habenula (LHab) shows strong activation that is restricted to animals having previously learned the association. Although intracellular mechanisms may be similar during consolidation and reconsolidation, this study indicates that different brain circuits are involved in the two processes, at least with respect to a rapidly learned olfactory task.
\end{abstract}

It is widely held that memory is represented in the brain by interactive networks that express experience-dependent plasticity. A major goal of research in neuroscience has been to describe such networks and understand the mechanisms of their plasticity. Cellular mechanisms of the synaptic remodeling underlying plasticity at a network level are beginning to be understood (Carew and Sutton 2001). Moreover, considerable progress has been made in delineating brain regions involved in different types of memory, for example, the hippocampus for spatial memory (O'Keefe and Speakman 1987), amygdala for memories based on emotional experiences (LeDoux 1996), and insular cortex for memories involving taste (Rosenblum et al. 1997). Finally, functional imaging studies in humans have indicated particular involvement of the frontal cortex and hippocampus in memory retrieval processes (Nyberg et al. 2000). Despite this progress, information concerning the interaction among different brain areas during formation and retrieval of an associative memory is relatively sparse and fragmented. Addressing this question requires simultaneously monitoring activity in multiple brain regions during learn-

${ }^{1}$ Corresponding author.

E-MAIL sjsara@ccr.jussieu.fr; FAX 33144273252.

Article and publication are at http://www.learnmem.org/cgi/doi/ $10.1101 / \mathrm{lm} .47802$. ing, the post-acquisition consolidation period, and retrieval and subsequent reconsolidation.

Ideally, a specific time stamp of when the learning actually takes place should be available to study post-acquisition consolidation processes. To meet this requirement, most studies rely on fear conditioning or passive avoidance, which can be learned in just a single trial. We have developed a rapidly learned, appetitively motivated odor-discrimination task that does not involve fear or acute stress. The rat merely pokes its nose into a hole in a sponge impregnated with the target odor to recover the reward. In just three trials, rats can learn to discriminate among three odors and associate one of them with a palatable reinforcement. The entire learning session takes just a few minutes, and the information is retained for at least $1 \mathrm{wk}$. This is in marked contrast to the usual odor-discrimination learning tasks requiring the learning of complex operant responses in many trials spaced over several sessions (Staubli et al. 1995; Hess et al. 1997; Datiche et al. 2001).

We used immunocytochemical marking by the immediate early gene c-fos to delineate specific brain regions activated during or after this associative learning and after reactivation of the well-consolidated memory by a retrieval test. Immediate early genes are supposed to act as messengers in coupling short-term neuronal activity with changes

LEARNING \& MEMORY 9:105-111 @ 2002 by Cold Spring Harbor Laboratory Press ISSN1072-0502/02 \$5.00

$$
\begin{array}{lllllllllllllllllllllllllll} 
& E & A & R & N & I & N & G & \& & M & E & M & O & R & Y
\end{array}
$$


at the level of gene transcription and as such should serve as markers by those neurons undergoing some modification as a result of experience (Sagar et al. 1988).

\section{RESULTS}

\section{Task Acquisition}

On the first trial, rats in the trained groups readily consumed the reinforcement visible on the edge of the sponge. It took as long as $4 \mathrm{~min}$ on the first trial for some rats to nose poke the correct sponge and consume the hidden reinforcement, but on the second trial, in which the reinforcement was not visible to the rat, there was a marked decrease in latency to find the correct sponge and make the nose-poke response and consume the hidden reinforcement. There were significant decreases in latency $(\mathrm{F}[4,40]=4.34$; $P=.001)$ and in the number of errors $(\mathrm{F}[4,40]=13.59$; $P<.0001)$ over trials. Rats rarely made a nose poke (error) to a nontargeted odor on the third trial, as can be seen in Figure 1. Unpaired control rats, each yoked to a trained rat for the time spent in the apparatus on each trial, readily consumed the reinforcement and showed approximately the same amount of exploratory activity during exposure to the apparatus and the odors as did their yoked trained partner.

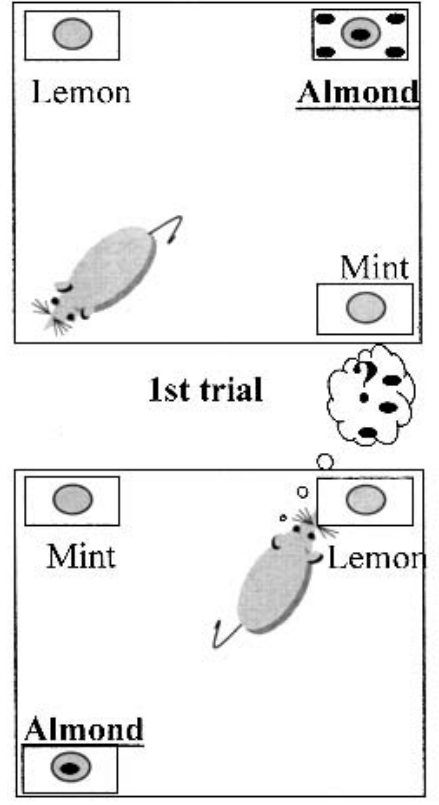

2nd trial

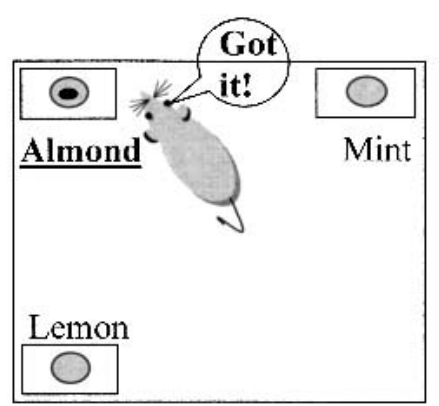

3rd trial

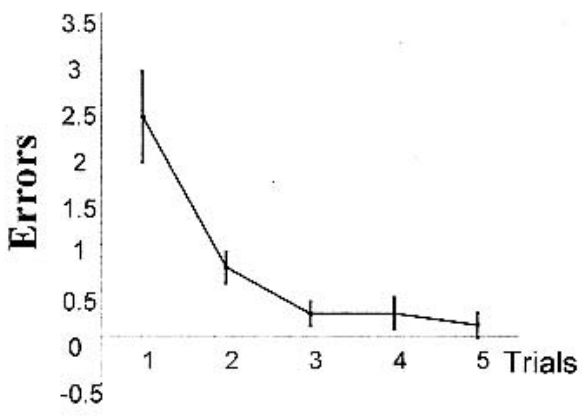

Figure 1 Odor task discrimination acquisition. Training was performed in a single session in five trials. On the first trial only, the reward was visible (almond, top left). The spatial configuration of the sponges was changed between trials and the reinforcement was always associated with the same odor. Trial $\times$ trial number of errors (nose poke to the nontarget odor) (mean \pm SEM) are shown on the graph. Similar results were obtained for latencies. This training procedure results in a long-term memory trace lasting at least $1 \mathrm{wk}$.

\section{c-fos Expression After Learning and After Retrieval}

Trained rats had visibly more immunolabeled cells than unpaired control rats in three regions: the prelimbic cortex (PLC), ventrolateral orbital cortex (VLO), and basolateral amygdala (BLA). An example of this is seen in Figure 2 showing the region of prefrontal cortex where there was more than a $100 \%$ increase in c-fos in trained rats compared controls; Figure 2, b, c, and d, shows immunoreactivity the brains of three different rats, one from each of the treatment groups, naive, unpaired, and trained, respecin most cases scores of 0 , precluded any quantitative analysis. Statistical analyses were applied to the data from trained and pseudotrained rats with separate $2 \times 2$ analyses of variance (ANOVAs) for each region.

\section{Frontal Cortex}

In PLC there was a significant main effect of the training condition $(\mathrm{F}[1,17]=22.01 ; P<.0002)$, a significant effect of time of sacrifice (after learning or after retrieval) $(\mathrm{F}[1,17]=23.96 ; P<.0001)$, and a significant interaction $(\mathrm{F}[1,17]=9.65 ; P<.006)$. Newman-Keuls pairwise comparisons confirmed that there were significantly more c-fos positive cells in the trained group killed immediately after training than in all other groups. In VLO there was an effect of training condition $(\mathrm{F}[1,17]=5.9 ; P<.02)$ and the time of sacrifice $(\mathrm{F}[1,17]=37.8$; $P<.0001)$; as seen in Figure 3 , there is a significant difference between the paired and unpaired groups only after training. In the infralimbic cortex (IL) region, there was the same pattern of results, but only the time of sacrifice factor was significant $(\mathrm{F}[1,17]=4.23 ; P=.05)$. Orthogonal comparisons showed that there were significantly more c-fos positive cells again in the trained group killed after training than in all the other groups. In the cingulate cortex (Cg1) there was a significant effect of the time of sacrifice factor $(\mathrm{F}[1,15]=40.47$; $P<.0001$ ), and pairwise comparisons revealed that there were significantly fewer c-fos positive cells in the paired group after retrieval than in the paired group after training.

\section{Amygdala}

The BLA showed marked increase in activation in the trained group, as can be seen in Figure 4 . There was a significant main effect of the training condition $(\mathrm{F}[1,13]=14.61$; $P<.002$ ), a significant effect of time of sac- 
a

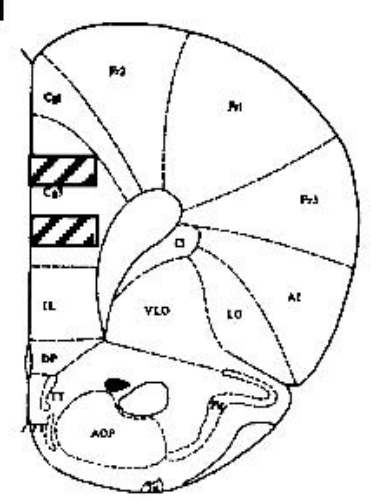

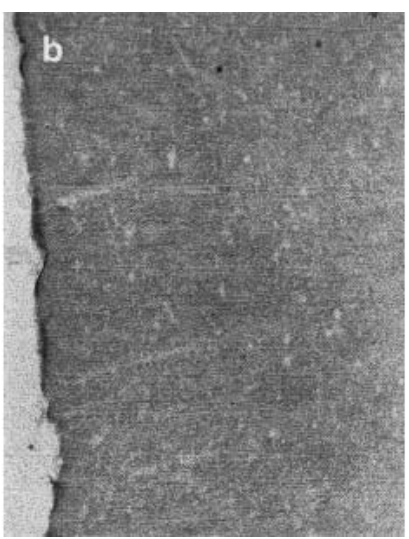
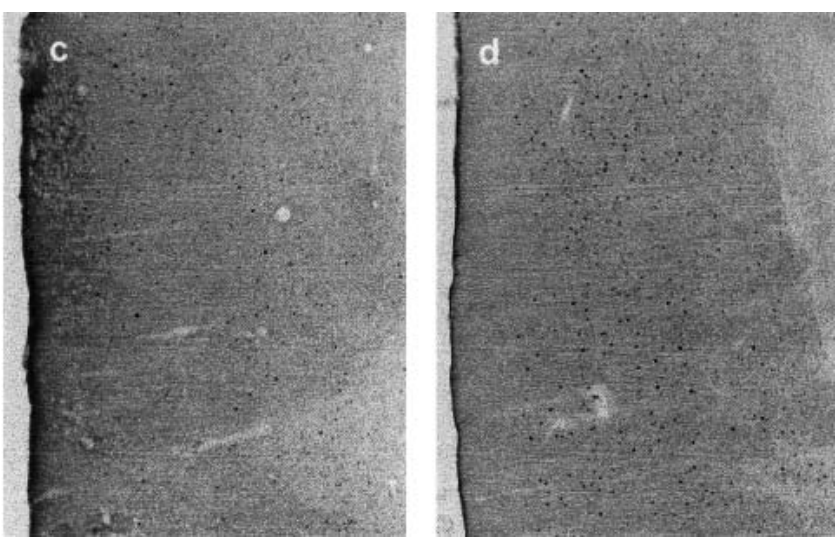

Figure 2 (a) Schematic diagram of a section taken from Paxinos and Watson (1986) showing the sampling regions within the prelimbic cortex (PLC) used for quantifying the number of marked cells. A computerized microscope stage that recorded X-Y coordinates ensured sampling from exactly the same place for each rat (see text). Immunoreactivity in the PLC is shown in naïve (b), unpaired yoked controls (c), and trained animals $(d)$. See text for treatment of each group. There are two visible marked cells in the naive rat $(b)$, relatively sparse marking in the yoked control $(c)$, and much denser marking in the trained rats $(d)$. Areas were delineated at the magnification shown in the figure; cells were counted at a higher magnification $(\times 40)$.

rifice $(\mathrm{F}[1,13]=35.21 ; P<.0001)$, and a significant interaction $(\mathrm{F}[1,13]=9.92 ; P<.007)$. Pairwise comparisons revealed that there were more c-fos positive cells in the trained group killed immediately after training than in all other groups. There were also fewer marked cells in the rats killed after retrieval than after training in both training conditions. There were no significant differences in the central amygdala (CeA).

\section{Piriform Cortex}

There were abundant fos-labeled cells in anterior piriform cortex (Cx Pir ant) and posterior piriform cortex (Cx Pir post) in both trained and unpaired groups, which was in

\section{Frontal regions}

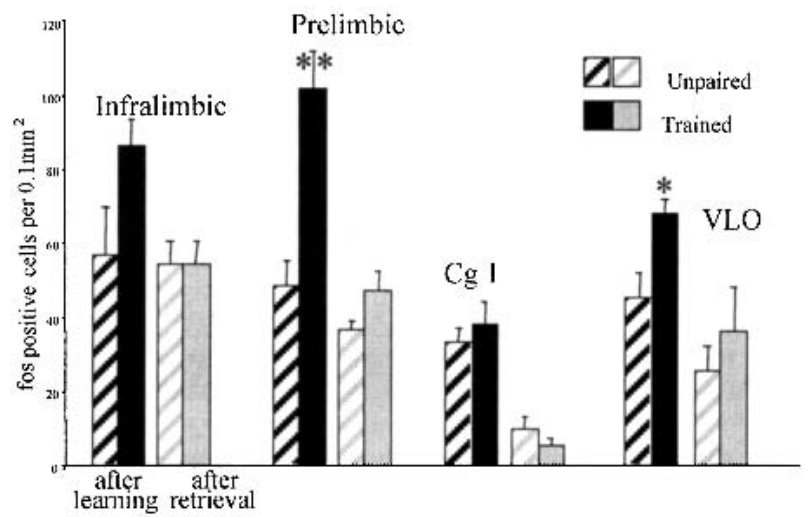

Figure 3 Frontal regions: Mean number of c-fos immunoreactive cells per $0.1 \mathrm{~mm}^{2}$ in frontal regions of unpaired controls and trained rats after training (left) or after retrieval test (right). In the $\mathrm{PLC}$ and ventrolateral orbital cortex (VLO), there was a significant increase in c-fos immunoreactivity in trained rats relative to controls after training (**prelimbic: $P<.005 ;{ }^{*}$ VLO: $\left.P=.03\right)$. marked contrast to naive quiet controls in which there were scarcely any marked cells in these regions. There was no difference in the intensity of the immunoreactivity between $\mathrm{Cx}$ Pir ant and Cx Pir post, and there were clearly no differences in trained and pseudotrained animals either after training or after the retrieval trial.

\section{Hippocampal Region}

In the hippocampus, there were only a few scattered immunoreactive cells in the CA1, CA3, or dentate gyrus (DG) in all groups, whereas in the neighboring perirhinal cortex (Prh), there was abundant labeling in all groups with no significant training-related differences.

\section{Habenula}

The lateral habenula (LHab) showed a modest number of

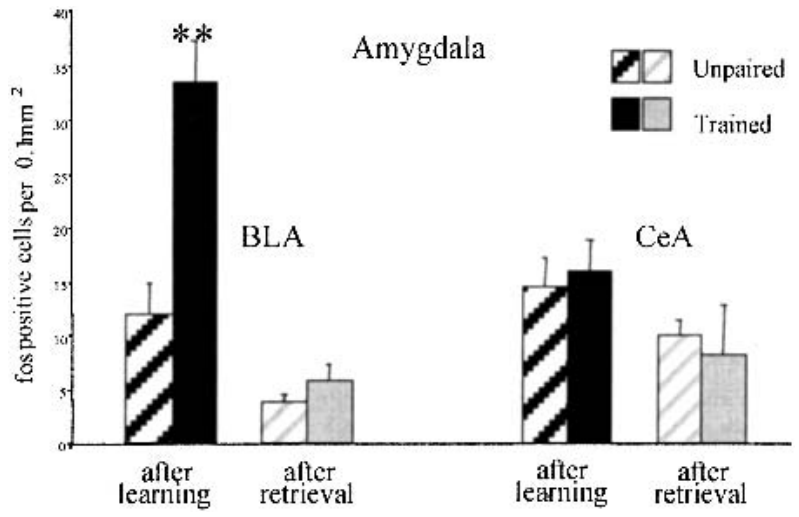

Figure 4 Amygdala: Mean number of c-fos immunoreactive cells per $0.1 \mathrm{~mm}^{2}$ in the basolateral amygdala (BLA) and the central amygdala $(\mathrm{CeA})$ of unpaired controls and trained rats after training (left) or after retrieval test (right). In the BLA, there was a significant increase in c-fos immunoreactivity in trained animals relative to controls after training. ( $\left.{ }^{* *} P<.005\right)$. There was not much difference in CeA after initial learning or retrieval and no effect of training in either condition.

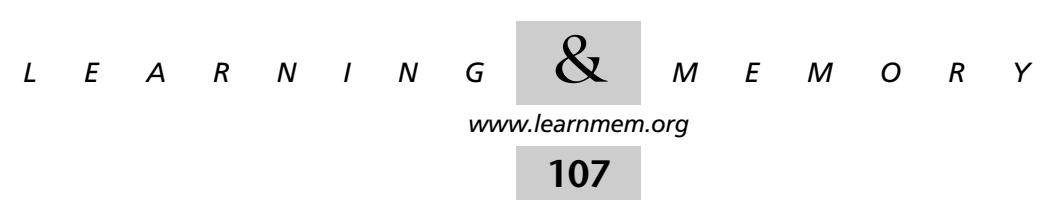


immunoreactive cells in both trained and unpaired controls after learning and after the retrieval test. ANOVA showed a significant effect of the training condition $(F[1,18]=8.09$; $P<0.01)$. Pairwise comparisons revealed that there were significantly more c-fos positive cells in the trained group killed after retrieval than in the corresponding pseudotrained group. No other pairwise comparisons were significant (Fig. 5). Immunoreactivity in the LHab from a representative trained rat killed after a retrieval test is shown in Figure 6a and its yoked control in Figure 6b. There is a total absence of marking in the medial portion, whereas c-fos marking is prominent in the LHab in the trained rat and sparse in the unpaired. Note that c-fos in the LHab was not significantly greater in trained rats compared with unpaired rats after the training session. c-fos marking in a rat killed after training is shown for comparison in Figure 6c.

\section{DISCUSSION}

The most striking result of this experiment is the very large difference in c-fos marking between trained and yoked unpaired control rats in limited and very specific regions, namely, PLC, VLO, and BLA. The simplicity of the task allows for relatively straightforward interpretation of the results. The only major difference between the trained rats and yoked controls was the association between the target odor and the reward. Several regions showed strong c-fos immunolabeling as a result of the handling, exploratory activity, and exposure to the odors and the chocolate-flavored rice breakfast cereal common to both groups, notably the primary olfactory cortices and the PRh. A recent study using an odor-discrimination task with more complex response requirements requiring extended training over several days

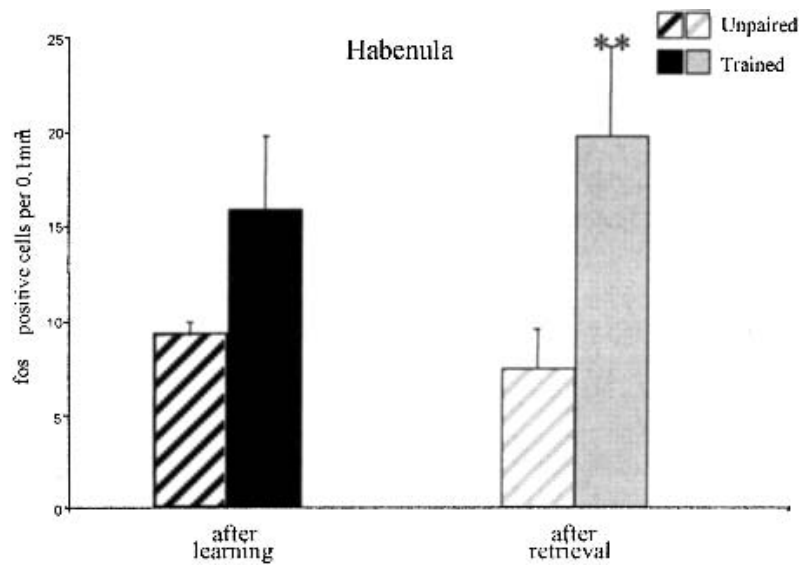

Figure 5 Habenula: Mean number of c-fos immunoreactive cells per $0.1 \mathrm{~mm}^{2}$ in the lateral habenula (LHab) of unpaired controls and trained rats after training (left) or after a retrieval test (right). This was the only region where there was a significant increase in c-fos immunoreactivity in trained animals relative to controls after a retrieval test $\left({ }^{* *} P=.03\right)$. There was no difference between trained and controls after the training session.
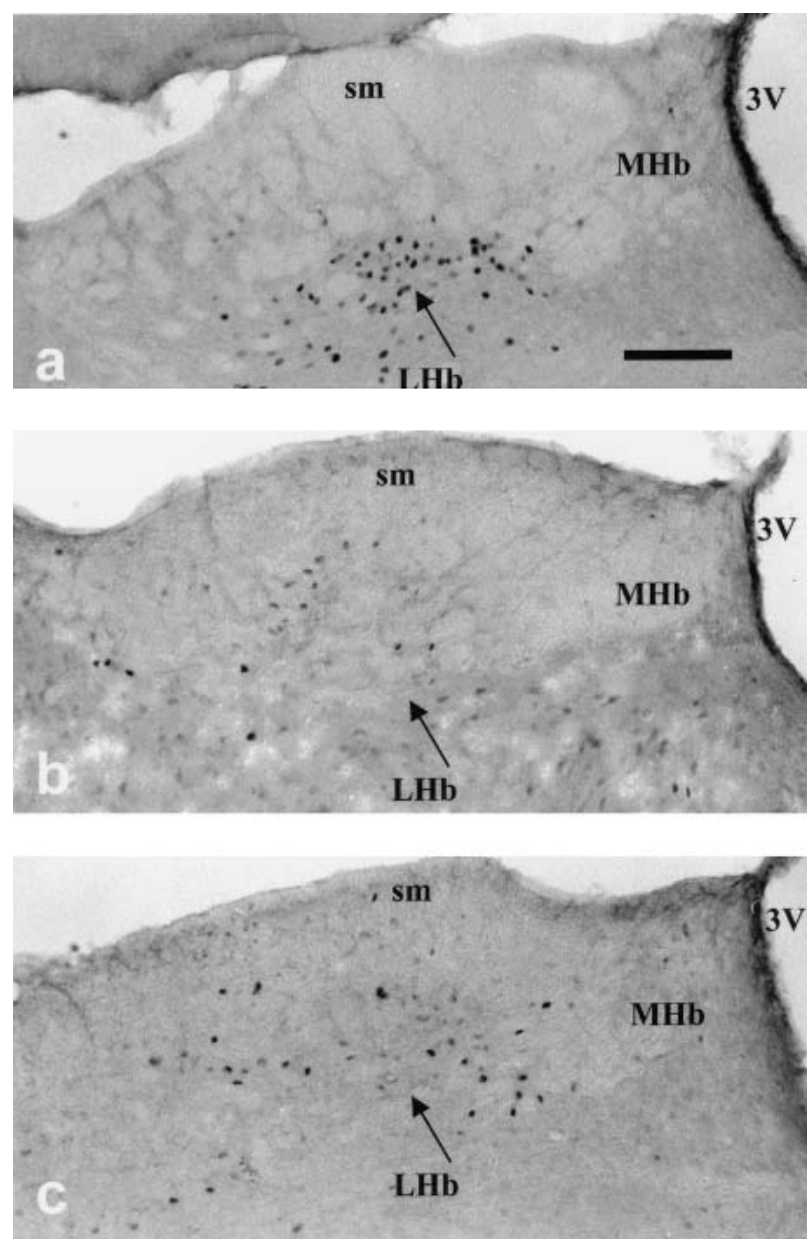

Figure 6 c-fos expression after learning or after retrieval in the LHab. (a) Section from a trained rat killed after the retrieval test. There are an abundant number of marked cells limited to the LHab. Note the absence of any c-fos immunoreactivity in the medial habenula. (b) Section from a yoked unpaired control rat after a 'retrieval' trial showing just a few scattered marked cells. (c) Section from a rat killed after initial training showing fewer and more scattered immunoreactive cells than in Figure 3a (scale bar, $100 \mu \mathrm{m}$ ).

also showed a nonspecific activation in c-fos in trained and pseudotrained rats compared with quiet controls. In those experiments, however, none of the regions examined showed differences between trained and pseudotrained controls (Datiche et al. 2001). This raises the all-important question of what constitutes an adequate control. In our experiments, rats are exposed to the odors and the sponges for the first time during the training, although they are familiar with the training environment and the reinforcement. Both trained and unpaired control groups have this new experience. Each group explores the environment and sniffs at the odors. The only difference in the treatment of the two groups is that one receives the reinforcement immediately before exposure to the discriminative stimuli, a backwards conditioning procedure, whereas the other group associates the target odor with the rein-

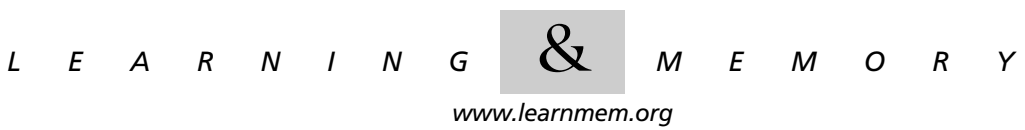


forcement. The duration of exposure of the unpaired control rats is determined by trial duration of the yoked partner. It is important to emphasize that there were no overt differences in locomotor activity, exploration, or sniffing at the sponges between the two groups during the training sessions. The learning that is different for the two groups is the specific odor-reward association. Thus it is not surprising that both groups show strong c-fos marking compared with naive controls in many brain regions associated with processing new information. Nevertheless, the rats learning the odor-reward association show a further robust activation in three specific brain regions.

Using in situ hybridization for c-fos message, Hess et al. (1997) showed selective activation of the BLA when the rat had to shift from free nose-poke responding to a go/no go odor discrimination contingency. They conclude that BLA is specifically activated by initial learning of the odor discrimination. Electrophysiological recording of neurons in this region during odor-discrimination learning confirms that BLA neurons rapidly develop responses to odors that are associated with reward (Schoenbaum et al. 1999). Moreover, in the same electrophysiological experiments, it was found that activity of neurons in the VLO region, where we also found specific learning-related c-fos, is related to the behavioral responses. Finally, recent electrophysiological data from our laboratory indicate that there is a population of neurons in the PLC of the frontal cortex that responds differentially to the discriminative odor stimuli during go/no go conditioning (Sara et al. 2002). Thus the three specific regions showing learning-specific c-fos immunoreactivity in the present experiment all have populations of neurons showing electrophysiological responses in odor-discrimination tasks.

c-fos immunoreactivity was conspicuous by its absence in all hippocampal subfields, indicating that the hippocampus was not highly activated during acquisition or posttraining consolidation. This non-engagement of the hippocampus in consolidation of odor memory should not be too surprising because hippocampal lesions do not induce deficits in simple odor-discrimination tasks (Staubli et al. 1995; Bunsey and Eichenbaum 1996). Furthermore, the results are compatible with our recent observation that injections of the NMDA (N-methyl-D-Aspartate) receptor antagonist APV (2-amino-5-phosphovaleric acid) directly into the hippocampus after training on this odor-discrimination task do not induce amnesia, whereas injections into either the cerebroventricles or the PLC region do (Tronel and Sara 2002). The lack of differences between the Cx Pir ant and Cx Pir post in both groups was surprising, because electrophysiological studies have provided strong evidence of functional dissociation of the two regions (the $\mathrm{Cx}$ Pir post being more involved in synaptic plasticity and memory processes than the Cx Pir ant). It should be noted, however, that those experiments used olfactory bulb stimulation as a condi- tioned stimulus, and extensive training lasting over several sessions (Litaudon et al. 1997; Mouly et al. 2001).

The lack of differences between trained and unpaired groups in c-fos marking on frontal regions after a retrieval trial is likewise quite surprising, because functional imaging studies in human (Nyberg et al. 2000) and nonhuman primates (Hasegawa 2000) have strongly implicated the prefrontal cortex in memory retrieval, particularly the initiation of retrieval processes. There are several possible explanations. Perhaps there is a real species difference, the rodent not using the frontal cortex to retrieve memory. There could be anatomical differences in brain regions engaged for retrieval according to the nature of the information to be retrieved. Moreover, the temporal dynamics of intracellular events triggered by retrieval may differ according to the cognitive demands of the task. There is, indeed, some evidence from our laboratory supporting this notion. Intracerebral ventricular injections of a beta receptor antagonist after reactivation of a multitrial spatial discrimination task is effective in inducing amnesia only at a time window at $1 \mathrm{~h}$ after the reactivation trial (Roullet and Sara 1998), whereas the same injection is effective at $2 \mathrm{~h}$ after the odor discrimination task (Sara et al. 1999). Only one time point for c-fos activation, $90 \mathrm{~min}$, was examined here.

It is possible that the PLC and VLO are activated during the initiation of retrieval of the association, but the activation is not of sufficient amplitude or duration to lead to induction of c-fos. On the other hand, another way of looking at the data is as a further control for the associative learning-dependent nature of the c-fos activation seen after training in specific regions. The rats killed after the retrieval test were already at an asymptotic level of performance and did not have to learn anything new. c-fos labeling after this experience is about the same as it is after the unpaired pseudotraining experience in frontal cortex. This is in marked contrast with what occurs in the BLA, where the c-fos labeling after the retrieval test indicates very little engagement of the amygdala after the initial learning takes place in the first training session (Fig. 5).

It should be kept in mind that the medial frontal cortex has direct synaptic input to the LHab (Greatrex and Phillipson 1982) where clear differential c-fos marking between trained and unpaired rats after retrieval was seen. Electrophysiological recording of neuronal activity simultaneously in these two regions after acquisition and after retrieval will shed further light on the role of the prefrontal cortex in activating the LHab during retrieval. The LHab receives converging information from the limbic forebrain, including the PLC and the extrapyramidal system, and thus should be an important integration site for organizing sensory-motor and affective information and response output (Sutherland 1982). Among target structures of LHab are the substantia nigra pars compacta $(\mathrm{SNc})$ and the ventral tegmental area (VTA) (Herkenham and Nauta 1979), both rich in dopamin-

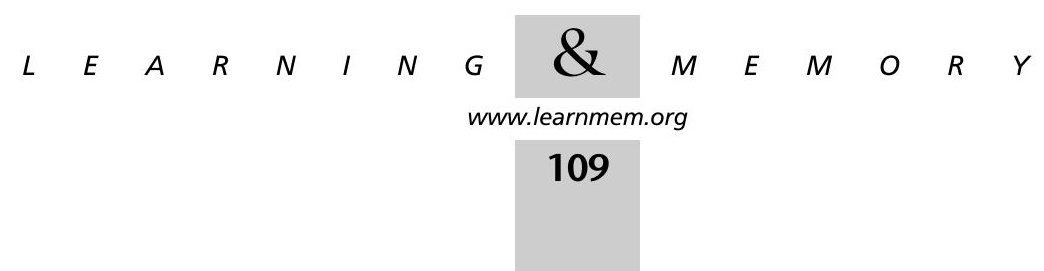


ergic neurons. Activation of LHab neurons appears to have a strong influence on these regions: Electrical stimulation of LHab suppresses activity of most putative dopamine neurons in SNc and VTA (Christoph et al. 1986). Although there is no direct projection of LHab to the noradrenergic nucleus locus ceruleus (LC), stimulation of LHab induces release of noradrenaline into forebrain areas, indicating that it activates the LC by some unknown pathway (Kalen et al. 1989). Thus, activation of the LHab at the time of memory retrieval, as indicated by the c-fos immunoreactivity in the present study, would have an important impact on neuromodulatory factors in forebrain regions. Regulation of noradrenaline (NA) and dopamine (DA) would be particularly important in the medial frontal cortex where the two modulators exert quite different influences on responses to sensory input (Mantz et al. 1988). The role of the LHab might be to provide an optimal balance between DA and NA for fine-tuning of processes underlying retrieval and promoting post-reactivation reconsolidation of memory (Sara 2000).

The present results showing associative learning-specific c-fos in the PLC, BLA, and VLO regions $90 \mathrm{~min}$ after rapid acquisition of an olfactory discrimination task indicates that these three anatomically related regions act in concert to consolidate memory about stimulus-response-reinforcement contingencies. Supporting this view are independent electrophysiological studies showing that these regions all have populations of neurons that show plasticity in firing during the learning session as a result of changes in the predictive value of the stimulus (Schoenbaum et al. 1999; Sara et al 2002). These experiments are the first to specify particular brain regions activated after associative learning as opposed to exploration of the environment, processing of sensory information, or organizing the response (Staubli et al. 1995; Hess et al. 1997; cf. Datiche et al. 2001). Moreover, some light has been shed on the question of whether the same neural circuits are implicated in reconsolidation processes as in the initial consolidation. The answer, at least for this simple associative learning in the rat, appears to be "no." The indication here of a role for the LHab in retrieving information concerning stimulus-reward associations is intriguing and indicates further study, especially in light of its anatomical and functional connections to both frontal cortex and brain stem neuromodulatory systems.

\section{MATERIALS AND METHODS}

\section{Animals}

Twenty-five Sprague Dawley rats, weighing between 200 and 220 $\mathrm{g}$, obtained from IFFA Credo, were housed in pairs in wire mesh cages. They were maintained on a 12-hr light-dark cycle. All rats were weighed and handled daily from the day of their arrival in the laboratory. There was free access to food and water except during the pre-training and training days when food was restricted to $\sim 15 \mathrm{~g}$ per rat per day to maintain body weight at $\sim 90 \%$ of freely feeding weight.

\section{Apparatus}

The training apparatus was a square box constructed of opaque plastic measuring $34 \times 34 \times 27 \mathrm{~cm}$. Sponges measuring $6 \times 7 \times 2$ $\mathrm{cm}$ deep had a hole of $2 \mathrm{~cm}$ in diameter cut into the center and were placed in glass side-holders of the same size. The food reinforcement was placed at the bottom of the opening in the sponge so the rat had to put its head inside the hole (nose poke) to obtain the reward of breakfast cereal. On the first trial only, four kernels of the breakfast cereal were also placed on the corners of that sponge impregnated with the target odor, as well as in the hole. The sponges with the nontargeted odor had empty holes. Sponges were placed in three corners of the box, and the position of each odor within the box was changed for each trial according to a previously determined protocol. The actual set of sponges was changed between trials as well to preclude any identification based on visual cues. Sponges were impregnated with an odor by placing $15 \mu \mathrm{L}$ of essence on each corner of the sponge. Discriminanda used were almond, mint, and lemon. Previous studies have indicated that rats have no systematic spontaneous aversion or attraction to any of these odors. In this experiment, almond was randomly chosen as the target odor. A videocamera was fixed above the apparatus, and the rat was observed on a video monitor in the same room. The sessions were recorded on videotape for possible reanalysis off-line.

\section{Experimental Design and Behavioral Procedures}

Rats were handled and weighed daily. The experiment began with $2 \mathrm{~d}$ of pre-training. The day before the first pre-training session, rats were mildly food restricted. The purpose of the pre-training was to familiarize the rats with the reinforcement and the experimental box. On the first day of the pre-training session, the rats were given free access to the chocolate-flavored rice breakfast cereal for 20 $\min$ in a neutral cage. The second day, rats were again allowed to consume the breakfast cereal for $10 \mathrm{~min}$ in the same neutral cage and then placed in the experimental box without the sponges for $10 \mathrm{~min}$.

Training was performed in a single session with more than five massed trials. The rat was introduced into the behavioral apparatus, in the corner without a sponge, head toward the wall. A 5-min ceiling time was imposed for the rat to find and consume the reinforcement in the hole of the sponge. Intertrial intervals were a minimum of $2 \mathrm{~min}$ and a maximum of $5 \mathrm{~min}$. The spatial configuration of the sponges was changed between trials and the reinforcement was always associated with the same odor. The latency for a correct response (nose poke into the reinforced sponge) and number of errors were recorded. Errors were counted as nose pokes into holes of sponges that contained odors other than the target odor or failure to nose poke after sniffing the sponge with the target odor. Most rats made no errors after the third trial, although the latencies to find the reward continued to decrease over the five trials. Unpaired controls $(n=5)$ were exposed to the reward immediately before the trial and then explored the apparatus containing the three odorous sponges, but no reinforcement, for the same amount of time as its yoked trained rat $(n=5)$. The pre-training was also the same as for the trained group. Thus unpaired rats received approximately equal exposure to all of the elements of the training with the reinforcement preceding the discriminative stimuli. For the reactivation, the rats were trained $(n=5)$ or pseudotrained in the unpaired procedure $(n=6)$ in exactly the same manner and received a retrieval trial $24 \mathrm{hr}$ after the training or pseudotraining. The retrieval trial consisted of one reinforced trial for the trained group and exposure for the same amount of time to reinforcement and odor in the experimental apparatus for the yoked unpaired

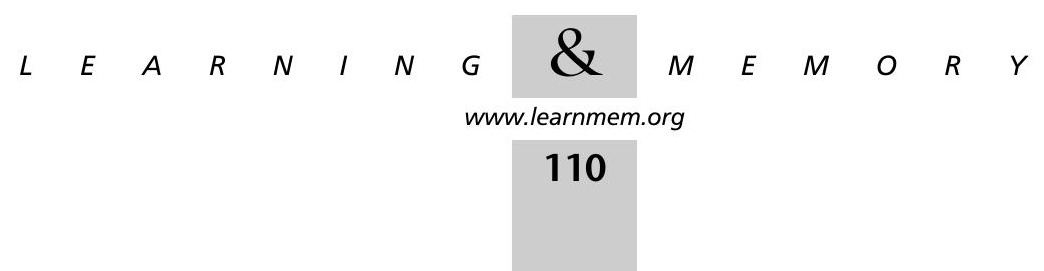


group. Home-cage control rats were used to determine baseline levels of c-fos expression. These rats were handled and weighed daily but were not food restricted $(n=4)$.

\section{Immunohistochemistry}

Rats were deeply anesthetized with pentobarbital $(60 \mathrm{mg} / \mathrm{kg}) 90$ min after the last trial. They were perfused transcardially with isotonic saline $(100 \mathrm{~mL}$ per rat) and a $4 \%$ paraformaldehyde solution ( $500 \mathrm{~mL}$ per rat). Brains were removed and post-fixed in the same fixative ( $4 \%$ paraformaldehyde) for $3 \mathrm{~h}$. After cryoprotection in phosphate buffer $(\mathrm{PB})$ containing $30 \%$ sucrose for $48 \mathrm{~h}$, the brains were cut coronally $(40 \mu \mathrm{m})$ with a cryostat and the sections collected in $0.1 \mathrm{M}$ phosphate buffer saline (PBS; pH 7.4). Free-floating sections were incubated for $5 \mathrm{~min}$ in PBS containing $3 \% \mathrm{H}_{2} \mathrm{O}_{2}$ and $10 \%$ methanol to eliminate endogenous peroxidase and then rinsed three times for $10 \mathrm{~min}$ in PBS. After a 15 -min incubation in $0.2 \%$ Triton X-100 in PBS, the sections were rinsed three times in PBS. These were placed in PBS containing 3\% BSA (bovine serum albu$\mathrm{min}$ ) for $1 \mathrm{hr}$, rinsed three times in PBS, and then incubated with the primary antibody overnight (c-fos $1: 500, \mathrm{sc}-52$ ) at $4^{\circ} \mathrm{C}$. After three rinses in PBS, the sections were incubated at room temperature for $2 \mathrm{hr}$ with the secondary biotinylated antibody (goat antirabbit) using the same dilution as the first antibody in PBS. After being washed, the sections were incubated for $90 \mathrm{~min}$ in avidinbiotin-peroxidase complex (ABC) solution. Then the sections were washed in PBS and twice in PB for 10 min each, placed in a solution of PB containing $0.1 \% 3,3^{\prime}$-diaminobenzidine (DAB; $10 \mathrm{mg} / 1 \mathrm{~mL}$ ), and developed by $\mathrm{H}_{2} \mathrm{O}_{2}$ addition $(0.02 \%)$. After processing, the tissue sections were mounted onto gelatin-coated slides and dehydrated through alcohol to xylene for light microscopic examination.

\section{Data Evaluation}

c-fos positive neurons were counted using a computerized image analyzer. Regions were carefully delineated, and marked cells were tagged and counted by an experimenter blind to treatment groups. Sections corresponded to two stereotaxic frontal planes $(3.2 \mathrm{~mm}$ anterior and $2.8 \mathrm{~mm}$ posterior to bregma, according to Paxinos and Watson [1986]). The regions included the following: the Cx Pir ant, Cx Pir post, Cg1, PLC, IL, VLO, medial agranular frontal cortex (FR2), BLA, (CeA), LHab, Prh, and three subfields of the hippocampus (CA1, CA3, and the dentate gyrus (DG). For each structure, labeled cells were counted within a predetermined sample region (Fig. 2a) chosen on the basis of the histological verification of adjacent sections stained with the cresyl violet. Cell counts were normalized for $\mathrm{mm}^{2} \times 10^{-1}$. A $2 \times 2$ ANOVA was used for statistical evaluation, with one factor being the training condition (paired or unpaired) and the other the time of sacrifice $(90 \mathrm{~min}$ after training or 90 min after retrieval test). Post hoc pairwise group comparisons were made by a Newman-Keuls test.

\section{ACKNOWLEDGMENTS}

We thank Y. Moricard for technical assistance and preparation of Figure 1. This work was supported by CNRS UMR 7102 .

The publication costs of this article were defrayed in part by payment of page charges. This article must therefore be hereby marked "advertisement" in accordance with 18 USC section 1734 solely to indicate this fact.

\section{REFERENCES}

Bunsey, M. and Eichenbaum, H. 1996. Conservation of hippocampal memory function in rats and humans. Nature 379: 255-257.

Carew, T.J. and Sutton, M.A. 2001. Molecular stepping stones in memory consolidation. Nat. Neurosci. 4: 769-771.
Christoph, G.R., Leonzio, R.J., and Wilcox, K.S. 1986. Stimulation of the lateral habenula inhibits dopamine-containing neurons in the substantia nigra and ventral tegmental area of the rat. J. Neurosci. 6: 613-619.

Datiche, F., Roullet, F., and Cattarelli, M. 2001. Expression of Fos in the piriform cortex after acquisition of olfactory learning: An immunohistochemical study in the rat. Brain Res. Bull. 55: 95-99.

Greatrex, R.M. and Phillipson, O.T. 1982. Demonstration of synaptic input from prefrontal cortex to the habenula in the rat. Brain Res. 238: 192-197.

Hasegawa, I. 2000. Neural mechanisms of memory retrieval: Role of the prefrontal cortex. Rev. Neurosci. 11: 113-125.

Herkenham, M. and Nauta, W.J. 1979. Efferent connections of the habenular nuclei in the rat. J. Comp. Neurol. 187: 19-47.

Hess, U.S., Gall, C.M., Granger, R., and Lynch, G. 1997. Differential patterns of c-fos mRNA expression in amygdala during successive stages of odor discrimination learning. Learn. Mem. 4: 262-283.

Kalen, P., Lindvall, O., and Bjorklund, A. 1989. Electrical stimulation of the lateral habenula increases hippocampal noradrenaline release as monitored by in vivo microdialysis. Exp. Brain Res. 76: 239-245.

LeDoux, J.E. 1996. The emotional brain. Simon and Schuster, New York

Litaudon, P., Mouly, A., Sullivan, R., and Cattarelli, M. 1997. Learning-induced changes in rat piriform cortex activity mapped using multisite recording with voltage-sensitive dye. Eur. J. Neurosci. 9: 1593-1602.

Mantz, J., Milla, C., Glowinski, J., and Thierry, A.M. 1988. Differential effects of ascending neurons containing dopamine and noradrenaline in the control of spontaneous activity and of evoked responses in the rat prefrontal cortex. Neuroscience 27: 517-526.

Mouly, A., Fort, A., Ben-Boutayab, N., and Gervais, R. 2001. Olfactory learning induces differential long-lasting changes in rat central olfactory pathways. Neuroscience 102: 11-21.

Nyberg, L., Habib, R., McIntosh, A.R., and Tulving, E. 2000. Reactivation of encoding-related brain activity during memory retrieval. Proc. Natl. Acad. Sci. 97: 11120-11124.

O'Keefe, J. and Speakman, A. 1987. Single unit activity in the rat hippocampus during a spatial memory task. Exp. Brain Res. 68: 1-27.

Paxinos, G. and Watson, C. 1986. The rat brain in stereotaxic coordinates. Academic Press, New York

Rosenblum, K., Berman, D.E., Hazvi, S., Lamprecht, R., and Dudai, Y. 1997. NMDA receptor and the tyrosine phosphorylation of its $2 B$ subunit in taste learning in the rat insular cortex. J. Neurosci. 17: 5129-5135.

Roullet, P. and Sara, S. 1998. Consolidation of memory after its reactivation: Involvement of beta noradrenergic receptors in the late phase. Neural Plast. 6: 63-68.

Sagar, S.M., Sharp, F.R., and Curran, T. 1988. Expression of c-fos protein in brain: Metabolic mapping at the cellular level. Science 240: 1328-1331.

Sara, S.J. 2000. Strengthening the shaky trace through retrieval. Nat. Rev. Neurosci. 1: 212-213.

Sara, S.J. et al. Neuronal activity in the medial prefrontal cortex and the locus coeruleus during go-nogo odor discrimination learning in the rat. Eur. J. Neurosci. Suppl. 12: (in press).

Sara, S.J., Roullet, P., and Przybyslawski, J. 1999. Consolidation of memory for odor-reward association: Beta-adrenergic receptor involvement in the late phase. Learn. Mem. 6: 88-96.

Schoenbaum, G., Chiba, A.A., and Gallagher, M. 1999. Neural encoding in orbitofrontal cortex and basolateral amygdala during olfactory discrimination learning. J. Neurosci. 19: 1876-1884.

Staubli, U., Le, T.T., and Lynch, G. 1995. Variants of olfactory memory and their dependencies on the hippocampal formation. J. Neurosci. 15: 1162-1171.

Sutherland, R.J. 1982. The dorsal diencephalic conduction system: A review of the anatomy and functions of the habenular complex. Neurosci. Biobehav. Rev. 6: 1-13.

Tronel, S. and Sara, S.J. NMDA receptors in prefrontal cortex but not hippocampus are necessary for memory formation of an odor-reward association. Eur. J. Neurosci. Suppl. 12: (in press).

Received March 4, 2002; accepted in revised form. April 30, 2002

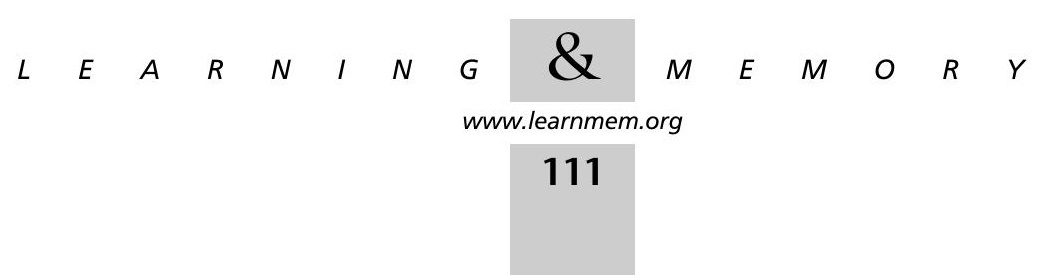




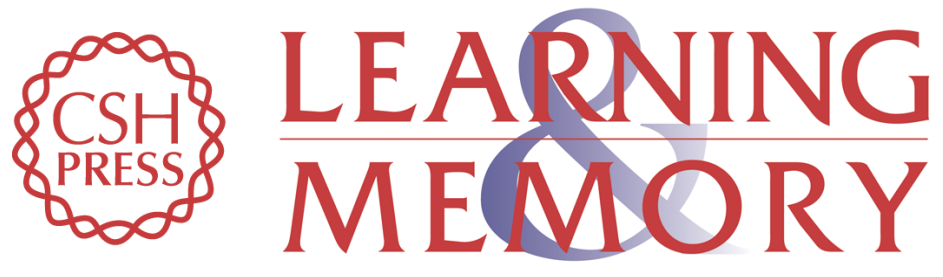

\section{Mapping of Olfactory Memory Circuits: Region-Specific c-fos Activation After Odor-Reward Associative Learning or After Its Retrieval}

Sophie Tronel and Susan J. Sara

Learn. Mem. 2002, 9:

Access the most recent version at doi:10.1101//m.47802

References This article cites 22 articles, 8 of which can be accessed free at: http://learnmem.cshlp.org/content/9/3/105.full.html\#ref-list-1

License

Email Alerting

Receive free email alerts when new articles cite this article - sign up in the box at the Service top right corner of the article or click here. 